(1)

CrossMark

\title{
Radiotherapy in the era of immunotherapy: teaching an old dog new tricks
}

\author{
B.C. John Cho ${ }^{1}{ }^{1}$ and Marc de Perrot $^{2}$
}

Affiliations: 'Dept of Radiation Oncology, Princess Margaret Cancer Centre, University Health Network, Toronto, ON, Canada. ${ }^{2}$ Division of Thoracic Surgery, Princess Margaret Cancer Centre, University Health Network, Toronto, ON, Canada.

Correspondence: B.C. John Cho, Dept of Radiation Oncology, OPG, 7-218, 700 University Av, Toronto, ON M5G 1Z5, Canada. E-mail: john.chodrmp.uhn.ca

@ERSpublications

Potentiating abscopal and vaccinal effects through combination immunoradiotherapy https://bit.ly/ 35Ewzv3

Cite this article as: Cho BCJ, de Perrot M. Radiotherapy in the era of immunotherapy: teaching an old dog new tricks. Eur Respir J 2020; 56: 2000320 [https://doi.org/10.1183/13993003.00320-2020].

The era of immunotherapy is revolutionising the treatment of solid tumours, as well as shifting the radiotherapeutic paradigm. Radiation that can boost the immune system, with non-ablative doses, provide new and exciting opportunities, such as the ability to generate an in situ vaccination. Specifically, we are interested in effecting a systemic (abscopal) response when combined with immunotherapy, and a durable (vaccinal) response when combined with surgery.

George Santayana, the American philosopher, once wrote "to know your future you must know your past." This year is the quasquicentennial of the X-ray's discovery. Radiation was employed as a cancer treatment almost contemporaneous to its discovery some 125 years ago. Wilhelm Conrad Röntgen discovered X-rays, serendipitously, while conducting cathode ray research in Würzburg, Germany, on November 8, 1895 [1]. In 1896, within a year of its discovery, in Chicago, USA, the first treatment was performed on a patient with recurrent breast cancer [2]. The rapid adoption of radiation as a cancer therapy, while remarkable, should not be viewed as confidence in its efficacy. Rather, it is a reflection of ineffective therapies and patient desperation.

Prior to the discovery of X-rays and chemotherapy, the only cancer treatment available was surgery. The therapy offered to the patient, back then, was based on the ablation paradigm: to cure the cancer, you must remove all the cancer cells. Although the concept of antisepsis was known through the earlier work of Ignaz Semmelweis, before the era of effective antibiotics and modern anaesthesia, patients undergoing invasive surgical procedures put their lives at risk, particularly from haemorrhage and infection. Cautery was one of the very few effective means to deal with these risks available back then. This essentially limited organs that could be surgically ablated to non-vital extremities that could be successfully cauterised, assuming the patient was able to avoid or overcome infection.

Non-invasive alternatives to surgery were, unsurprisingly, eagerly sought. Radiation had several attractive physical properties that made it suitable as a replacement for surgery. Most importantly, electromagnetic radiation is completely immaterial (massless) and, thus, able to penetrate matter, non-invasively. As later discovery and experiment will show, radiation is composed of high energy photons: quantum mechanical packets of energy. They are electrically neutral, unlike protons and electrons, so they tend to interact less with matter compared to their charged counterparts and, thus, penetrate more deeply as they deposit energy in tissue (dose). 
Subsequent research discovered some important radiobiologic properties: more radiation dose killed more cancer cells; when divided into smaller daily doses (fractionation), radiation had a differential effect with relative sparing of slowly proliferating tissue (such as connective tissue and muscle). Reducing toxicity was of particular advantage since normal tissue is often admixed within the irradiated target volume.

Radiation oncology is a medical specialty that has seen tremendous technological advancements, such as treatment planning systems for radiation planning and linear accelerators for radiation delivery. Technology has sufficiently advanced that we are reaching the physical limits of what is possible in terms of dosimetric precision. For example, high precision paraspinal radiotherapy deals with planning organ at risk margins of the order of $1.5 \mathrm{~mm}$, which is smaller than the physical penumbra of high energy photons (typically 3-5 mm). This level of dosimetric precision requires comparable dedication to millimetre-level rigor and precision throughout the entire planning and delivery process and represents the very limit of what is currently achievable.

Tremendous success has been achieved following the ablative paradigm. As radiotherapy becomes more and more ablative, the clinical trajectory is becoming more and more like surgery. We are approaching local control rates comparable to surgical lobectomy with lung stereotactic ablative radiotherapy (SABR) [3]. Yet, we may be approaching the radiotherapeutic limits of the ablative paradigm in the sense that SABR suffers the same constraints and limitations as surgical ablation; namely, certain structures cannot be ablated without significant morbidity. Serially organised structures are functionally dependent on previous subunits and intolerant to localised damage which can cause dysfunction downstream (such as the spinal cord or oesophagus), exactly like the broken weak link in a chain. Just as surgery is not recommended for bulky mediastinal nodes, lung SABR is not recommended if the cytotoxic dose overlaps central, mediastinal structures.

Having reached the current limits, in attempting to answer which direction to point to next, it is helpful to remember Satayana's words earlier. Perhaps by applying some general principle as evidenced from the past, we can predict or, at least, constrain the future. If we look back, we can see that enhancing selectivity improves the therapeutic index. All advances in the ablative paradigm follow the principle of selectivity. From this perspective, treatment is a "clinical sieve" that identifies and eliminates the tumour (target) from the normal tissue (background) by improving the discrimination between target and background. All technical improvements are judged on how well they maximise the positives (efficacy) and minimise the negatives (toxicity) in addressing the problem of target identification and elimination. For example, surgery has evolved from pneumonectomy with mass ligation of the hilum staged with a chest X-ray to anatomical segmentectomy staged with an endobronchial ultrasound and positron emission tomography-computed tomography. In this general sense, improving selection involves improving specificity (better normal tissue sparing and less toxicity) and/or improving sensitivity (less target underdosing and better efficacy).

As we near the current technical limits of radiotherapy (millimetre level), we will not achieve smaller scales and tighter tolerances without some new technical breakthrough or paradigm shift. In principle, we would like to be able to treat down to the sub-millimetre or micrometre level (which is approaching the scale of individual cells).

The dream of immunotherapy is directing an immune response against tumour-specific antigens, resulting in effective systemic (abscopal) and durable (vaccinal) response [4]. We have performed laboratory experiments to investigate radiation-mediated abscopal response leading to an "out-of-field" effect. We have shown this effect to be immune-mediated in that it is extinguished in immunocompromised mice [5]. We have also demonstrated an in situ vaccination with rejection of tumour rechallenges several months after treatment of the first tumour with short courses of non-ablative radiation followed by surgery [6]. The in situ vaccination effect is enhanced when the initial tumour is completely removed, unleashing the full potential of the immune effect.

There is burgeoning interest and growing evidence looking at combining radiotherapy and immune checkpoint inhibitors [7]. Our experiments suggest that one of the major barriers to in situ vaccination and the abscopal effect is the overall tumor volume and its immunosuppressive effects potentially mediated by cancer stem cells resistant to treatment [8]. Hence, the full benefit of the immune activation by non-ablative radiation could potentially be obtained in early stage disease as long as the visible primary tumour is completely resected or ablated.

How best to implement this strategy in early stage disease is still unclear. Intervening in early stage disease with non-ablative radiotherapy and immune checkpoint inhibitors in association with surgery, aside from utilising all three treating modalities, would provide the advantage of leveraging the patient's own immune system. This has evolved to be highly selective with maximal efficacy and minimal/no toxicity, which are 
the very same properties sought in cancer therapy. Successful treatment depends on complex interplay between diverse host, disease and treatment factors. For example, the best outcomes result when patients are young and fit, when cancers are small, localised and indolent. Large, aggressive, extensive cancers need more aggressive, toxic treatment. These are, naturally, associated with more failures, more recurrences, and more morbidity. Oligofractionated (3-5 fractions) radiotherapy appears to help potentiate the immune response [9].

However, the optimal radiotherapy dose, fractionation and treatment sequencing needed to potentiate immune response are still not fully understood. We hypothesise that the optimal ("sweet spot") radiotherapy dose cannot be too high (excessively immunosuppressive) nor too low (not sufficiently immunogenic) and that different (sub)volumes and organs may react differently to different doses of radiotherapy with the ultimate aim of a durable, systemic, tumoricidal response. Thus, the optimal dose distribution may not be uniform, as per traditional (i.e. International Commission on Radiation Units) approach, but instead, non-uniform. We are currently studying heterogeneous oligofractionated ("hotshot") radiotherapy followed by surgical resection to test this.

As treatment advances, the complexity increases. Rather than viewing cancer treatment as sequential steps amongst different modalities, the modern view is more holistic where different modalities interact, resulting in superior overall clinical outcomes and less overall toxicity. The body's own immune system is unrivalled in identifying and eliminating foreign invaders, par excellence. Recent immunotherapy trials $[10,11]$ have dramatically changed the therapeutic landscape. The ultimate hope being, by leveraging the host's immune system with deft, gentle, clinical "nudges", one might vaccinate oneself against the cancer.

Conflict of interest: B.C.J. Cho has nothing to disclose. M. de Perrot reports personal fees for advisory board work from Merck, personal fees for lectures from Bayer, outside the submitted work.

\section{References}

1 Röntgen W. Ueber eine neue Art von Strahlen. Vorläufige Mitteilung. Aus den Sitzungsberichten der Würzburger Physik.-Medic. Gesellschaft Würzburg 1895: 137-147.

2 Grubbe EH. X-ray treatment; its introduction to medicine. J Am Inst Homeopath 1946; 39: 419-422.

3 Senan S, Paul MA, Lagerwaard FJ. Treatment of early-stage lung cancer detected by screening: surgery or stereotactic ablative radiotherapy? Lancet Oncol 2013; 14: e270-e274.

4 Sharabi AB, Lim M, DeWeese TL, et al. Radiation and checkpoint blockade immunotherapy: radiosensitisation and potential mechanisms of synergy. Lancet Oncol 2015; 16: e498-e509.

5 Wu L, Wu MO, De la Maza L, et al. Targeting the inhibitory receptor CTLA-4 on T cells increased abscopal effects in murine mesothelioma model. Oncotarget 2015; 6: 12468-12480.

6 De La Maza L, Wu M, Wu L, et al. In situ vaccination after accelerated hypofractionated radiation and surgery in a mesothelioma mouse model. Clin Cancer Res 2017; 23: 5502-5513.

7 Ngwa W, Irabor OC, Schoenfeld JD, et al. Using immunotherapy to boost the abscopal effect. Nat Rev Cancer 2018; 18: 313-322.

8 Wu L, Blum W, Zhu CQ, et al. Putative cancer stem cells may be the key target to inhibit cancer cell repopulation between the intervals of chemoradiation in murine mesothelioma. BMC Cancer 2018; 18: 471.

9 Wang Y, Deng W, Li N, et al. Combining immunotherapy and radiotherapy for cancer treatment: current challenges and future directions. Front Pharmacol 2018; 9: 185.

10 Antonia SJ, Villegas A, Daniel D, et al. Durvalumab after chemoradiotherapy in stage III non-small-cell lung cancer. N Engl J Med 2017; 377: 1919-1929.

11 Horn L, Mansfield AS, Szczesna A, et al. First-line atezolizumab plus chemotherapy in extensive-stage small-cell lung cancer. N Engl J Med 2018; 379: 2220-2229. 